\title{
Thermodynamics of the anisotropic Heisenberg chain calculated by the density matrix renormalization group method
}

\author{
Naokazu Shibata \\ Institute for Solid State Physics, University of Tokyo, 7-22-1 Roppongi, Minato-ku, Tokyo 106, Japan
}

\begin{abstract}
The density matrix renormalization group (DMRG) method is applied to the anisotropic Heisenberg chain at finite temperatures. The free energy of the system is obtained using the quantum transfer matrix which is iteratively enlarged in the imaginary time direction. The magnetic susceptibility and the specific heat are calculated down to $T=0.01 \mathrm{~J}$ and compared with the Bethe ansatz results. The agreement including the logarithmic correction in the magnetic susceptibility at the isotropic point is fairly good.
\end{abstract}

The density matrix renormalization group (DMRG) method has brought significant progress to the field of numerical calculations. This method provides a way to study long chains by iteratively enlarging the system size and to obtain the ground state wave function with only small systematic errors [1]. Although the DMRG method was initially designed for one-dimensional quantum systems it has also been applied to two-dimensional classical systems [2], and now even to two-dimensional quantum systems [3]. A recent advance in the use of the DMRG method is its application to thermodynamics $4,5,5$. In the present paper, we apply the DMRG method to the quantum transfer matrix and obtain accurate results which allow us to study low temperature behaviors of thermodynamic quantities such as the logarithmic correction in the magnetic susceptibility of the Heisenberg chain.

Initially, the DMRG procedure at finite temperature was designed to be a similar algorithm to the zero temperature DMRG except that in the former we take several relevant low-energy states as target states to obtain a representation of the Hamiltonian most efficiently at a given temperature [1,5]. By adding the density matrices, calculated from the low-energy states using a normalized Boltzmann weight, one can construct an effective density matrix and find important bases by diagonalizing it. While this method was successfully applied to finite systems, its application to the infinite system is difficult because, in general, we need to increase the size of the system while decreasing the temperature, which introduces an enormous truncation error to the calculation.

To deal with the infinite system in a more systematic way, one can use the transfer matrix method. In this method the free energy of the infinite system is directly obtained from the maximum eigenvalue of the transfer matrix [6,7]. The partition function of the system whose Hamiltonian $H$ is decomposed into two parts $H_{\text {odd }}=\sum_{n=1}^{L / 2} h_{2 n-1,2 n}$ and $H_{\text {even }}=\sum_{n=1}^{L / 2} h_{2 n, 2 n+1}$ such as $\left[h_{2 n-1,2 n}, h_{2 n^{\prime}-1,2 n^{\prime}}\right]=\left[h_{2 n, 2 n+1}, h_{2 n^{\prime}, 2 n^{\prime}+1}\right]=0$ is represented by the transfer matrix $\mathcal{T}$ as

$$
\begin{aligned}
Z & =\operatorname{Tr} \mathrm{e}^{-\beta H} \\
& =\lim _{M \rightarrow \infty} \operatorname{Tr}\left[\left(\mathrm{e}^{-\beta H_{\text {odd }} / M} \mathrm{e}^{-\beta H_{\text {even }} / M}\right)\right]^{M}
\end{aligned}
$$

$$
=\operatorname{Tr} \prod_{n=1}^{L / 2} \mathcal{T}_{n}
$$

where $\mathcal{T}_{n}$ is defined by

$$
\mathcal{T}_{n}=\lim _{M \rightarrow \infty}\left[\mathrm{e}^{-\beta h_{2 n-1,2 n} / M} \mathrm{e}^{-\beta h_{2 n, 2 n+1} / M}\right]^{M} .
$$

Thus, once we know the maximum eigenvalue $\lambda_{\max }$ of $\mathcal{T}_{n}$, we can get the partition function

$$
Z=\lambda_{\max }^{L / 2}
$$

when $\mathcal{T}_{n}$ is translationally invariant and the length of the system $L$ is large enough.

The problem now, is how to calculate $\lambda_{\max }$ of $\mathcal{T}$, the matrix size of which diverges with increasing $M$. To manage this difficulty we use the DMRG method [4]: We iteratively increase $M$ in $\mathcal{T}$ by restricting the number of bases used in $\mathcal{T}$ and keeping $\lambda_{\max }$ optimal. For this purpose we define $\mathcal{T}^{A}(M=2)=\mathrm{e}^{-\beta_{0} h_{\text {odd }}}$ and $\mathcal{T}^{B}(M=2)=\mathrm{e}^{-\beta_{0} h_{\text {even }}}$ with $h_{\text {odd }}=h_{2 n-1,2 n}$ and $h_{\text {even }}=h_{2 n, 2 n+1}$, and increase $M$ of $\mathcal{T}^{A(B)}(M)$ as

$$
\begin{aligned}
\mathcal{T}^{A}(M) \mathrm{e}^{-\beta_{0} h_{\text {even }}} & \rightarrow \mathcal{T}^{A^{\prime}}(M+1) \\
\mathrm{e}^{-\beta_{0} h_{\text {odd }}} \mathcal{T}^{B}(M) & \rightarrow \mathcal{T}^{B^{\prime}}(M+1), \\
\mathcal{T}^{A^{\prime}}(M) \mathrm{e}^{-\beta_{0} h_{\text {odd }}} & \rightarrow \mathcal{T}^{A}(M+1) \\
\mathrm{e}^{-\beta_{0} h_{\text {even }}} \mathcal{T}^{B^{\prime}}(M) & \rightarrow \mathcal{T}^{B}(M+1) .
\end{aligned}
$$

The optimal set of bases at the transformations in eq. (6) will be determined as follows. We first diagonalize $\mathcal{T}=$ $\mathcal{T}^{A} \mathrm{e}^{-\beta_{0} h_{\text {even }}} \mathrm{e}^{-\beta_{0} h_{\text {odd }}} \mathcal{T}^{B}$ with the periodic boundary conditions in the $\beta$ direction, and obtain left and right eigenvectors for $\mathcal{T}, V^{L}$ and $V^{R}$, which correspond to the maximum eigenvalue of $\mathcal{T}$. We then compose the density matrix $\rho_{i i^{\prime}}^{A}$ for the bases of $\mathcal{T}^{A} \mathrm{e}^{-\beta_{0} h_{\text {even }}}$ as

$$
\rho_{i i^{\prime}}^{A}=\sum_{j} V_{i j}^{L} V_{i^{\prime} j}^{R}
$$

where $i$ and $j$ are indices of the bases for $\mathcal{T}^{A} \mathrm{e}^{-\beta_{0} h_{\mathrm{even}}}$ and $\mathrm{e}^{-\beta_{0} h_{\text {odd }}} \mathcal{T}^{B}$ as shown in Fig. 1 . The best way to restrict the number of bases is to find a proper transformation to new bases $i \rightarrow i_{\text {new }}$ which maximize the norm 
$\sum_{i j} V_{i j}^{L} V_{i j}^{R}$, with a fixed number $m$ of $i_{\text {new }}$. To do this we use the left and right eigenvectors of the $m$ largest eigenvalues $\gamma^{i \text { new }}$ of the $\rho_{i i^{\prime}}^{A}$ to be the transformation matrix to the new bases $i_{\text {new }}$. The error accompanied by this truncation is defined by $1-\sum_{i_{\text {new }}=1}^{m} \gamma^{i \text { new }}$.

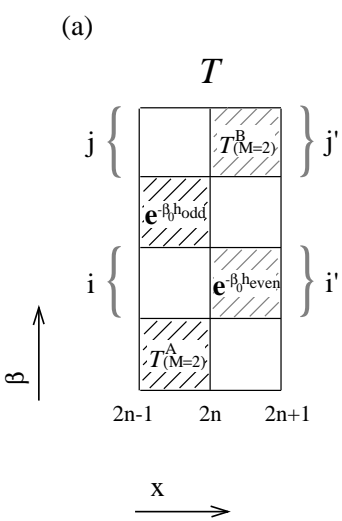

(b)

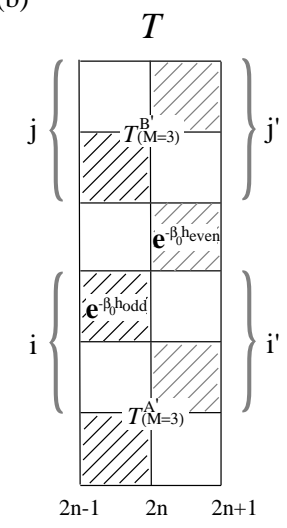

FIG. 1. (a) Transfer matrix used at the renormalization in eq. 6. $(M=2)$ (b) Transfer matrix used at the renormalization in eq. 8 . $(M=3)$

The most significant difference of the DMRG procedure for the transfer matrix from the zero-temperature DMRG algorithm is that, in the former, we have to use the non-symmetric density matrix. This leads to the left and right eigenvectors of the density matrix, $u_{i}^{i \text { new }}$ and $v_{i}^{i}{ }^{i}$ ew , being different. For this reason we have to transform the transfer matrix using these vectors as

$$
\mathcal{T}_{i_{\text {new }} i_{\text {new }}^{\prime}}^{A}=\sum_{i i^{\prime}} v_{i}^{i_{\text {new }}} \mathcal{T}_{i i^{\prime}}^{A} v_{i^{\prime}}^{i_{\text {new }}^{\prime}}
$$

where $v_{i}^{i \text { new }}$ and $u_{i}^{i_{\text {new }}}$ satisfy the orthogonality

$$
\sum_{i} v_{i}^{i_{\text {new }}} u_{i}^{i_{\text {new }}^{\prime}}=\delta_{i_{\text {new }} i_{\text {new }}^{\prime}}
$$

Technically, the non-symmetric nature of the density matrix is a difficult point of the numerical calculation. In fact, if we do not pay attention to the number of states kept, the eigenvalue of the density matrix becomes complex. In order to avoid this we fix the number states in the density matrix throughout the calculation.

In the following we apply the above DMRG method to the $S=1 / 2$ anisotropic Heisenberg chain to check the reliability of the method. The Hamiltonian is given by

$$
H=J \sum_{i}\left\{S_{i}^{x} S_{i+1}^{x}+S_{i}^{y} S_{i+1}^{y}+\Delta S_{i}^{z} S_{i+1}^{z}\right\}
$$

where $\Delta$ is the anisotropy parameter which changes the interaction from XY type to Ising type with increasing
$\Delta: \Delta=0$ corresponds to the $\mathrm{XY}$ limit, and $\Delta=1$ the isotropic point. For this model it is well known that there are three different regimes. When $\Delta>1$ a finite gap exists in the excitation spectrum and both the magnetic susceptibility and the specific heat show exponential decay at low temperatures. At $\Delta=1$, the excitation gap closes, and the system shows a critical behavior with a logarithmic correction in $\chi$ at low temperatures [8]. In the region $\Delta<1$, the logarithmic correction in $\chi$ disappears and $\chi$ approaches a finite value in accordance with a power law. Thus this model provides a rich variety of structures in the thermodynamic quantities and is an appropriate test case for the present method.

In this calculation we use the simple power method to obtain the maximum eigenvalue and corresponding eigenvector of the non-symmetric transfer matrix, and use the lapack library to diagonalize the non-symmetric density matrix. The maximum number of states $m$ in the density matrix is 76 and the corresponding truncation error is $5 \times 10^{-5}$ at the lowest temperature $T=0.01 \mathrm{~J}$. In order to carry out the extrapolation for the Trotter number $M$ we start the DMRG procedure from several initial temperatures $\beta_{0}$ and iteratively enlarge the transfer matrix in the imaginary time direction using the infinite system algorithm with decreasing temperature.

We first show magnetic susceptibility $\chi^{z}$ obtained by the present method. $\chi^{z}$ is determined from the change in the free energy $\delta F=\chi^{z} h^{2} / 2$ in a small magnetic field $h=0.01 J$ along the z-axis. The calculated $\chi^{z}$ in the XY limit is shown in Fig. 2(a). The exact result is also plotted in the figure [9]. The agreement with the exact result is excellent even at the lowest temperature $T=0.01 \mathrm{~J}$. It should be mentioned that such a quadratic behavior in $\chi^{z}$ is difficult to reproduce if we use a simulation based on a finite system where $\chi^{z}$ always vanishes exponentially or diverges in the low temperature limit. The result at $\Delta=0.5$ is presented in Fig. 2(b). Similarly to Fig. 2(a), $\chi^{z}$ decreases to a finite value with a power law with decreasing temperature. The obtained power law exponent is 2.0 which is consistent with the result of the bosonization [8]. The isotropic case is presented in Fig. 2(c). In this case the result of the Bethe ansatz is also plotted for comparison. The rather rapid decrease in the low temperature region is due to the logarithmic correction. The small discrepancy at $T=0.01 \mathrm{~J}$ is considered to originate from the finite nature of the magnetic field and from truncation errors in the DMRG. An Ising like case $(\Delta=2.0)$ is presented in Fig. 2(d). In this case $\chi^{z}$ approaches zero with an exponential form consistent with the existence of an excitation gap. The estimated value of the gap is consistent with the exact value $0.15 J$ [10]. 


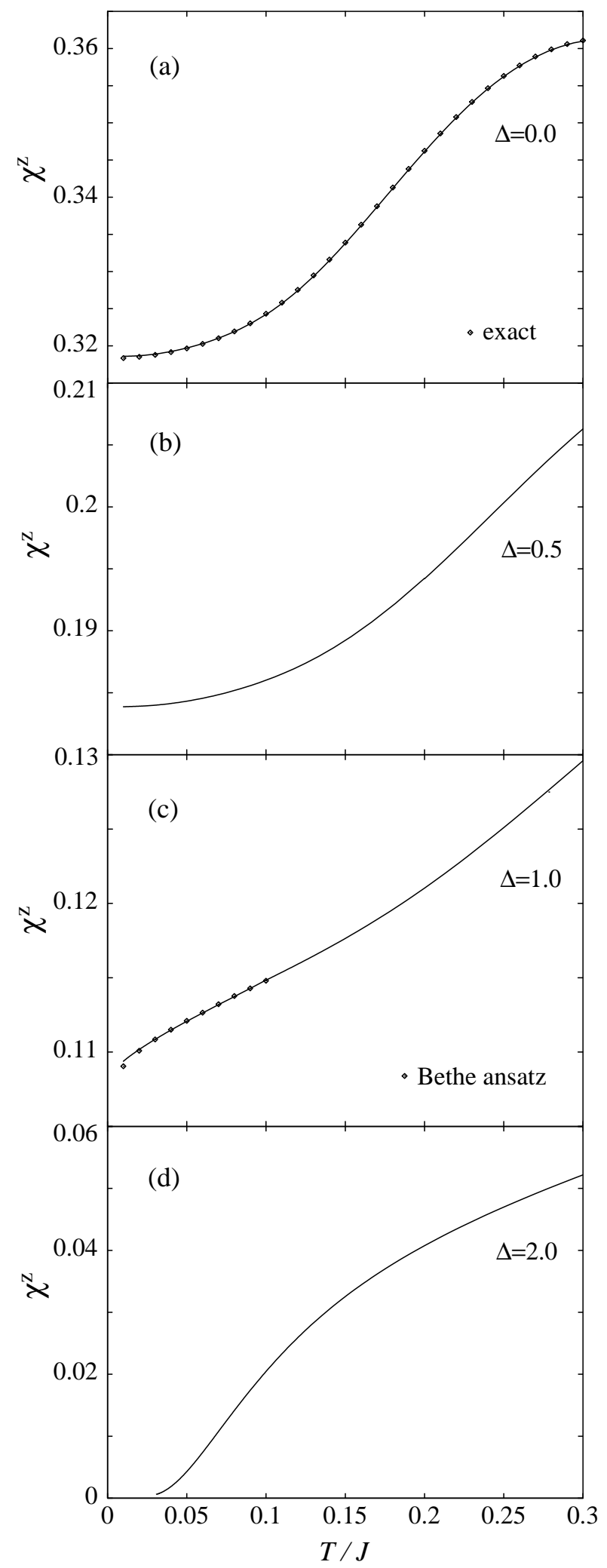

FIG. 2. Magnetic susceptibility of the $\mathrm{S}=1 / 2$ Heisenberg chain calculated by the DMRG.

We next calculate the specific heat. The specific heat is determined by the second derivative of the free energy; $C=-T \partial^{2} F / \partial T^{2}$. The results for $\Delta=0.0,0.5,1.0$, and
2.0 are shown in Fig. 3. The exact results in the XY limit $(\Delta=0)$ are also plotted. In the XY-limit the DMRG results reproduce the exact values well. For $\Delta \leq 1.0$ the specific heat vanishes linearly as $T$ approaches zero. For $\Delta=2.0$, on the other hand, it seems to be almost zero at finite $T$ consistent with the existence of the spin gap. Thus all the results obtained by the present DMRG calculation are consistent with the expected behaviors.

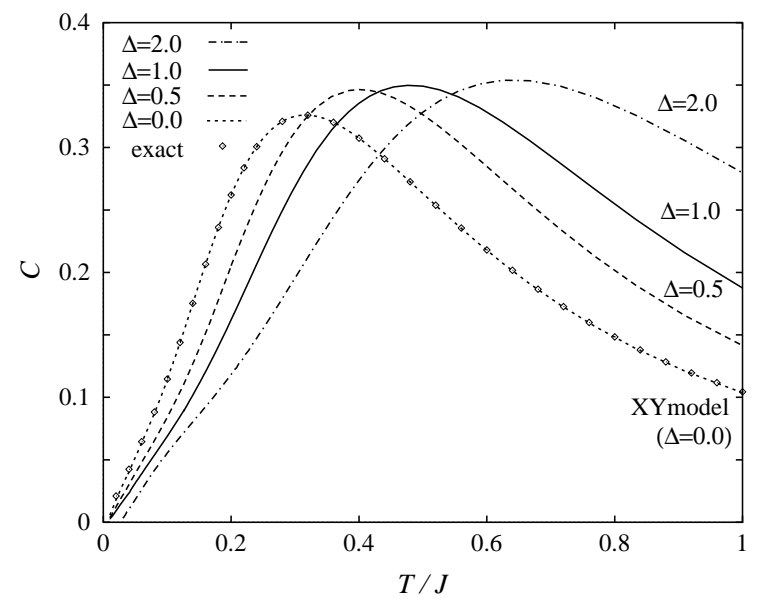

FIG. 3. Specific heat of the $\mathrm{S}=1 / 2$ Heisenberg chain calculated by the DMRG

To summarize, we have shown that the DMRG method at finite temperature is formulated using the transfer matrix and that this method actually provides reliable results even at low temperatures. The magnetic susceptibility and the specific heat of the Heisenberg chain are accurately calculated for both cases in which the excitation spectrum is gapless and gapfull. The logarithmic correction in the magnetic susceptibility is correctly reproduced at the isotropic point where the system shows the highly critical behavior. Since this method can be applied to both fermion systems and frustrated spin systems, the DMRG method at finite temperatures will be a promising numerical method with which to study the low temperature properties of one-dimensional quantum systems.

During the present calculation the author was informed that X. Wang and T. Xiang have done similar work [11]. The author would like to thank Beat Ammon, Manfred Sigrist, Matthias Troyer, and Kazuo Ueda for valuable discussions. Numerical calculations were performed on VPP500 at the ISSP, University of Tokyo. The author is supported by the Japan Society for the Promotion of Science. 
[1] S. R. White: Phys. Rev. Lett. 69 (1992) 2863; Phys. Rev. B 48 (1993) 10345.

[2] T. Nishino: J. Phys. Soc. Jpn. 64 (1995) 3598.

[3] S. R. White: Phys. Rev. Lett. 77 (1996) 3633.

[4] R. J. Bursill, T. Xiang, and G. A. Gehring: J. Phys. Condns. Matter 8 (1996) L583.

[5] S. Moukouri and L. G. Caron: Phys. Rev. Lett. 77 (1996) 4640.

[6] H. Betsuyaku: Phys. Rev. Lett. 53 (1984) 629; Prog. Theor. Phys. 73 (1985) 319.

[7] T. Koma: Prog. Theor. Phys. 81 (1989) 783.

[8] S. Eggert, I. Affleck, and M. Takahashi: Phys. Rev. Lett. 73 (1994) 332.

[9] S. Katsura: Phys. Rev. 127 (1962) 1508; 129 (1963) 2835.

[10] A. Virosztek and F. Woynarovich: J. Phys. A 17 (1984) 3029.

[11] X. Wang and T. Xiang: cond-mat/9705301. 